\title{
INTEGRATION OF MAINTENANCE CONSTRAINTS IN SCHEDULING: FUZZY MODELLING AND MULTI-AGENT APPROACH
}

\author{
Thierry Coudert, Bernard Grabot, Bernard Archimède \\ $L G P / E N I T$ \\ 47, Avenue d'Azereix - BP 1629 \\ F-65016 TARBES Cedex \\ FRANCE \\ e-mail: \{coudert, bernard, archi\}@enit.fr
}

The scheduling problem should be solved through a negotiation process: negotiation between functions of the companies, or between companies in the case of networked enterprises. We show in this paper how a multi-agent approach can allow to build a schedule which provides a compromise between the partially conflicting objectives of several services, namely Production, Sales and Maintenance. Some of the considered constraints are not crisp in an industrial context and have been modelled using fuzzy temporal windows in order to bring the conflict solving technique closer to the industrial reality.

\section{INTRODUCTION}

Sales, maintenance, inventory control or manpower management are closely linked with production at the operational level of the manufacturing process, even if their respective objectives can be partially conflicting. As a consequence, performing a schedule should be regarded as a multi-objective process allowing to balance the objective satisfaction of all the concerned functions. Nevertheless, the schedule usually only considers production constraints, and the obtained result acts as a framework within which the other functions have to try to satisfy their own objectives. This behaviour implicitly considers that Production is the leading function, even if it only results from the inability of the planning tools to support negotiation. Similarly, several networked enterprises can be involved in the manufacturing of a product. The manufacturing operations can be either scheduled at the level of the leading company, the others having to adapt themselves to this framework, or can be negotiated in order to integrate constraints coming from different entities. In order to develop scheduling tools adapted to this negotiation context, multi-agent systems have appeared as much promising during the last years. The multi-agent paradigm is based on the principle of negotiation between agents 
having different objectives: it provides so a "natural" way for solving problems through compromises and has often been applied to scheduling (see e.g. (Sohier et al., 1998), (Saad et al., 1996), (Schäfer et al., 1996) or (Tranvouez et al., 1998)). We show in this paper how a multi-agent system may allow to involve various entities in the scheduling process, with the example of compromises between Production, Sales and Maintenance.

In that purpose, we have taken the RAMSES multi-agent scheduling system (Archimède and Coudert, 1998) as a basic framework. RAMSES allows to define several entities involved in the scheduling process with their own objectives: a multi-agent system representing the Maintenance interests has been developed, and a general negotiation protocol between Production, Sales and Maintenance has been suggested (Coudert et al., 1999). This paper describes how the definition of fuzzy temporal windows can help to better take into account the maintenance, production and sales degrees of freedom in the negotiation process.

\section{A MULTI-AGENT SCHEDULING SYSTEM DEALING WITH MAINTENANCE AND MANUFACTURING OPERATIONS}

\subsection{The RAMSES environment}

The RAMSES environment has been developed in order to test various scheduling strategies in a multi-agent context. The RAMSES multi-agent model is based on the architecture described in Fig. 1.

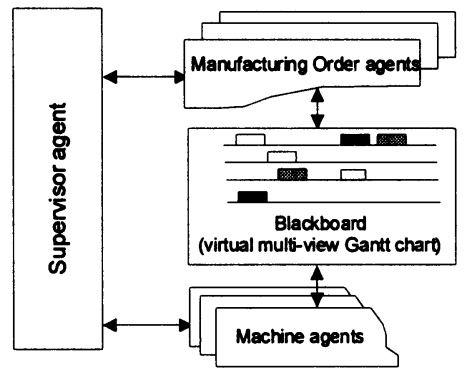

Figure 1 - RAMSES architecture

At the beginning of the scheduling process, the supervisor agent creates and initialises the manufacturing order (MO) and machine agents. It is then in charge of controlling the execution of the negotiation cycles between agents and the access to the blackboard describing the current solution and the bids under negotiation. A MO agent has to plan manufacturing operations defined in its associated routing. Each operation of the routing corresponds to an activity (drilling, milling, etc.) which can be performed by several machines with a different efficiency. The possible objectives of a MO agent are to minimise its cycle time, to respect a due date or to minimise a manufacturing cost (since the machines have different hourly rates). 
Each MO agent expresses its requirements by a bid containing the operations and their characteristics (activity, minimum processing times). This bid consists in an earlier infinite capacity planning which is put on the blackboard. This blackboard can be seen as a virtual Gantt chart representing the current state of the negotiation process at different conceptual levels: operations are first associated to activities, then to machines and some operations are definitively located whereas others can still be moved. The Machine agents make bids on the operations offered by the MO agents. These bids consist in correcting the processing time according to their capacity to perform the activity, and pushing the operation to the date where they can perform it. Two different bids are made by each Machine agent: one gives a possible position taking into account all the operations that the machine can get (effective position) while the other is made as if the considered operation is the only one that will be performed (potential position). Each MO agent compares the different bids received from the machine agents to its objectives. The MO agents can accept the effective positions suggested by the machine agents: the negotiation process is then over. If the effective position does not give satisfaction, and if the potential position is much better, the MO agent can take the risk to wait, expecting that this potential position will become effective. In that purpose, it makes a new bid on the base of the potential position.

This type of communication between agents through bids is close to the Contract Net Protocol (Smith, 1990) which has often been applied to scheduling, like in (Saad et al., 1996). The major difference is that an order is usually planned operation by operation without any competition with the other orders whereas in RAMSES, manufacturing orders are competing with each other for each operation. In the first case, the processing sequence of the manufacturing orders has a great influence on the result. The concept of effective and potential position used in RAMSES also gives new opportunities to improve a first possible solution through time.

\subsection{Integration of Maintenance agents in the RAMSES environment}

In order to take into account the objectives of the Maintenance function, we have defined a new agent category: the Maintenance agents. A Maintenance agent is associated to each Machine agent, and is in charge of planning the preventive maintenance activities on a machine. A Maintenance agent has a list of maintenance activities, together with the corresponding temporal windows that describe their optimal temporal location. As it is done in industrial applications so that in research studies ((Brandolese et al., 1996), (Sanmarti et al., 1997) we suppose that each temporal window has been defined by making a compromise between the maintenance cost (which increases if maintenance activities are often performed) and the machine reliability, which decreases when the time between two maintenance activities increases. In the same way, a temporal window can be defined for a manufacturing operation, like in analysis under constraints (Erschler et al., 1976). In that case, the window of the $\mathrm{n}^{\text {th }}$ manufacturing operation is defined by placing the previous operations of the routing as early as possible, and the following ones as late as possible. The time between the end of operation n-1 and the beginning of operation $n+1$ defines the temporal window of operation $n$.

The negotiation process between a Machine agent and a Maintenance agent is defined as follows: for each new manufacturing operation to be scheduled by a 
Machine agent, this agent will ask its Maintenance agent whether this operation can be in conflict with a maintenance activity. If there is a conflict, the Maintenance agent checks whether it is possible to move the maintenance operation inside its temporal window. If the end of the Maintenance operation remains inside the window, the Maintenance agent accepts the change and the Machine agent validates the operation. Otherwise, the Maintenance agent asks the Machine agent whether it is possible to move the manufacturing operation in its own temporal window and suggests an ending date. If it is possible, the operation is re-planned, otherwise it is necessary to find a compromise by relaxing either the maintenance or the manufacturing temporal constraints. This negotiation process is summarised in Figure 2.

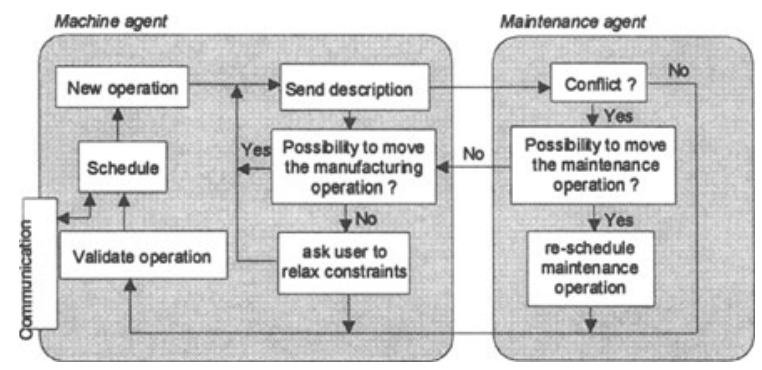

Figure 2 - Co-operation/negotiation between Maintenance and Machine agents

This version of the system, of course called RAMSES II, has been successfully tested (Coudert et al., 1999). Nevertheless, the definition of temporal windows as crisp intervals is quite far from the industrial reality where the location of a maintenance or manufacturing operation is mainly a matter of preference. We show in next section how fuzzy logic can help to define in a unique formalism the temporal constraints and their possible degrees of relaxation in order to allow a real negotiation process.

\section{DEFINITION OF FUZZY TEMPORAL WINDOWS}

The idea of modeling relaxable temporal constraints by fuzzy sets has often been used in scheduling, from (Kerr and Walker, 1989) to (Ishii, 2000). We shall see in the following sections how fuzzy temporal windows can be defined for due dates, manufacturing operations and maintenance operations.

\subsection{Fuzzy modeling of a due date}

A fuzzy due date is defined here as described in Fig. 3: the order may be early (full satisfaction, i.e. $\mu=1$ ) and a delay becomes progressively unacceptable (satisfaction from $\mu=1$ to $\mu=0$ for the last possible finishing date). On Fig. 3, the last manufacturing operation is represented by a hachured rectangle: on that example, the satisfaction of the due date constraint is 0.3 : it becomes a matter of degree instead of a binary value. 


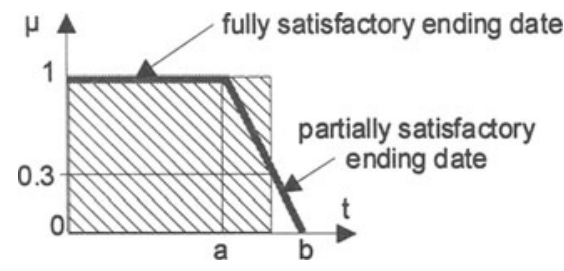

Figure 3 - Fuzzy modelling of a due date

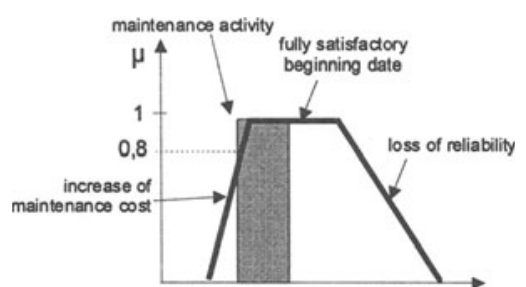

Figure 4 - Preferred beginning dates for a maintenance activity

\subsection{Fuzzy modeling of a Maintenance operation}

The fuzzy set of Fig. 4 describes the possible beginning of a maintenance activity. Starting from the centre, the left slope can be interpreted as a decreasing satisfaction due to an early maintenance that increases the maintenance cost. The right slope can be interpreted as a decreasing satisfaction due to a possible loose of reliability. This degree of satisfaction is e.g. 0.8 for the activity of Fig. 4 .

\subsection{Fuzzy modeling of a manufacturing operation}

The fuzzy temporal window of a manufacturing operation is calculated when the operation has to be planned, by analogy with crisp ones as they are described in section 2.2. The support of the fuzzy set (i.e. the points for which $\mu \neq 0$ ) is the period of time between the first possible beginning of the operation (place (1) on Fig. 5) and the last possible beginning, i.e. with the operation is set at the latest possible position, denoted (2). In order to determine what positions give complete satisfaction, we have chosen to share the slack time between the operations to be planned (considered operation + remaining ones). On Fig. 5, three operations (hachured rectangles) plus the considered one (white rectangle) remain to be planned. The slack time is divided by four, and the postionning of the manufacturing operation to be planned is considered as giving full satisfaction if the operation only consumes its part of the slack time. After position (2), it will not be possible to meet the due date anymore, so this position will be considered as providing a null satisfaction (see Fig. 5). If the due date is itself fuzzy, the crisp due date of Fig. 5 is replaced by the last point of the fuzzy due date. If it is not possible to meet the due date (i.e. position (1) = position (2) and (end of last operation > due date)), we consider that only an immediate beginning of the operation gives satisfaction: the fuzzy temporal window is then reduced to a point.

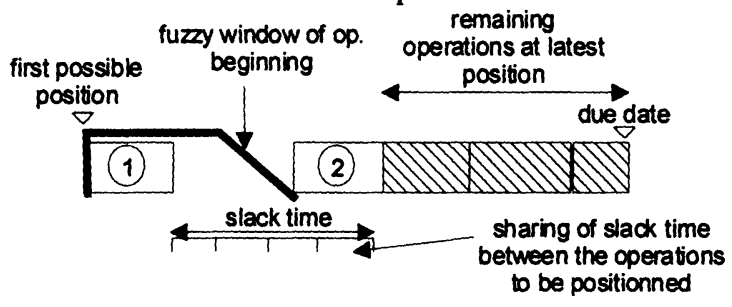

Figure 5-Fuzzy temporal window for the beginning of a manufacturing operation 
A conflict solving method allowing to deal with these degrees of freedom is described in next section.

\section{CONFLICT SOLVING}

With the notation illustrated in Fig. 6, the condition of conflict between a manufacturing and a maintenance operation is that $(\mathrm{b} 1+\mathrm{L} 1>\mathrm{c} 2)$ AND $(\mathrm{b} 2+\mathrm{L} 2>\mathrm{c} 1)$. Various criteria may be used to express the compromise between the satisfaction degree of the maintenance and manufacturing operations (e.g. maximising the average degree of satisfaction, the minimum one, etc.). The latter has here been chosen but other criteria may be defined in order to express more complex relationships between maintenance and production.

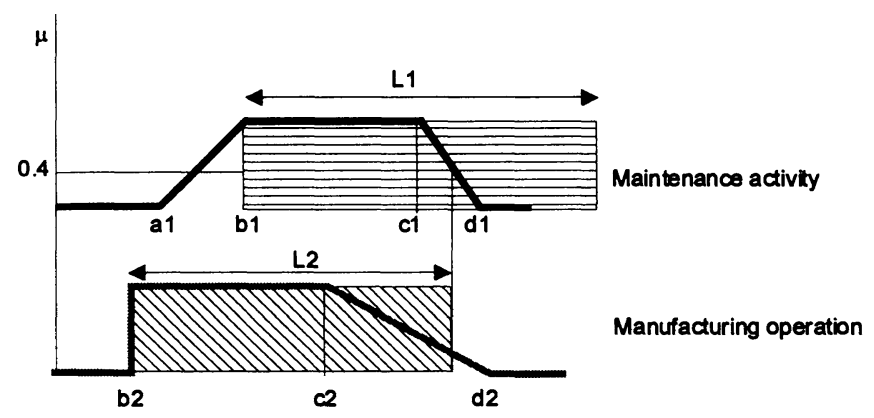

Figure 6 - Conflict between a maintenance activity and a manufacturing operation

We can see on Fig. 6 that:

- the manufacturing operation can be performed first without having a null satisfaction of the maintenance activity position if $(\mathrm{b} 2+\mathrm{L} 2<\mathrm{d} 1)$. In that case, the optimal position is a manufacturing operation performed as early as possible (beginning at b2) followed by the maintenance activity beginning at (b2+L2). The position of the manufacturing operation gives then satisfaction with a degree 1 , whereas the position of the maintenance activity gives satisfaction with a degree 0.4.

- the maintenance activity can be performed first without having a null satisfaction of the manufacturing operation position if $(\mathrm{a} 1+\mathrm{L} 1<\mathrm{d} 2)$. Since we want to maximise $\min (\mu$ (begin ManOp), $\mu$ (begin MaintAct)), the best position is obtained when $\mu$ (begin ManOp) $=\mu$ (begin MaintAct). The maintenance activity begins in the ascending front of the fuzzy set at the top of Fig. 6 or in its kernel, whereas the manufacturing operation begins in the kernel of the fuzzy set at the bottom of Fig. 6 or in its descending front.

If the two conditions are satisfied $((\mathrm{b} 2+\mathrm{L} 2<\mathrm{d} 1)$ and $(\mathrm{a} 1+\mathrm{L} 1<\mathrm{d} 2))$, the two previous solutions must be tested (maintenance first or production first) in order to choose the positions that provide the best satisfaction degrees. If none of the two conditions is satisfied, one of the positions will be satisfied with a degree 1 , and the other with a degree 0 . It is so necessary to ask the user whether the maintenance or the production will be privileged. 


\section{EXAMPLES OF OBTAINED SCHEDULE}

The fuzzy temporal windows and the conflict management hereby described have been implemented in RAMSES II. An example of result is shown on Fig. 7 with 25 manufacturing orders with fuzzy due dates on two machines on which 9 maintenance activities (white hachured rectangles $(1$ to 9 ) must be planned. Only the fuzzy windows of the maintenance activities and their associated satisfaction degree are shown since the fuzzy window of a manufacturing operation is dynamic and is difficult to show on the resulting Gantt. Maintenance activities 3, 0, $\boldsymbol{0}$ and $\boldsymbol{\Theta}$ have been postponed and maintenance activity $\boldsymbol{\Theta}$ has been planned earlier during the negotiation process, aiming at maximizing the satisfaction degrees of both maintenance activities and manufacturing operations. For the maintenance activities $\boldsymbol{0}, \mathbf{2}, \boldsymbol{5}$ and $\boldsymbol{8}$, no conflict was detected and they have been planned with a satisfaction degree of 1 .

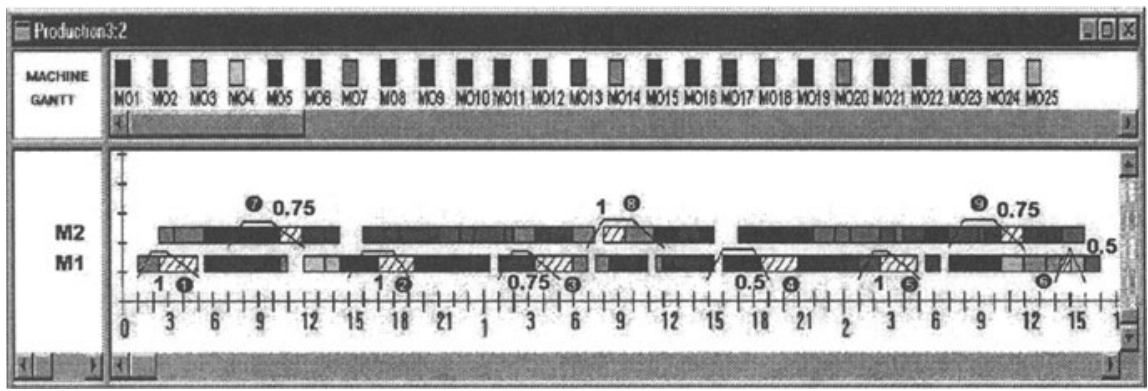

Figure 7 - Example of schedule

Three strategies of co-operation have been tested on this example: priority to production (manufacturing operations are planned first and next maintenance activities), priority to maintenance (maintenance activities are planned first) and finally the negotiation process described above. Fig. 8 shows the assessment of results. It represents the percentage of manufacturing orders and maintenance activities with regard to their satisfaction degrees. The negotiation process provides a better compromise between production and maintenance constraints and better respect of manufacturing orders due dates than the two other solutions.

\section{CONCLUSION}

The scheduling process should be considered as the result of a negotiation between functions within the company, or even between companies in the case of networked enterprises. We have shown in this paper that fuzzy logic may provide a way to model the degrees of freedom of the negotiation in a quite natural way, with an illustration on a negotiation between production and maintenance. New developments are in progress using the RAMSES II software in order to explicitly 
consider the co-ordination of distant workshops and the constraints implied by the supply chain management.

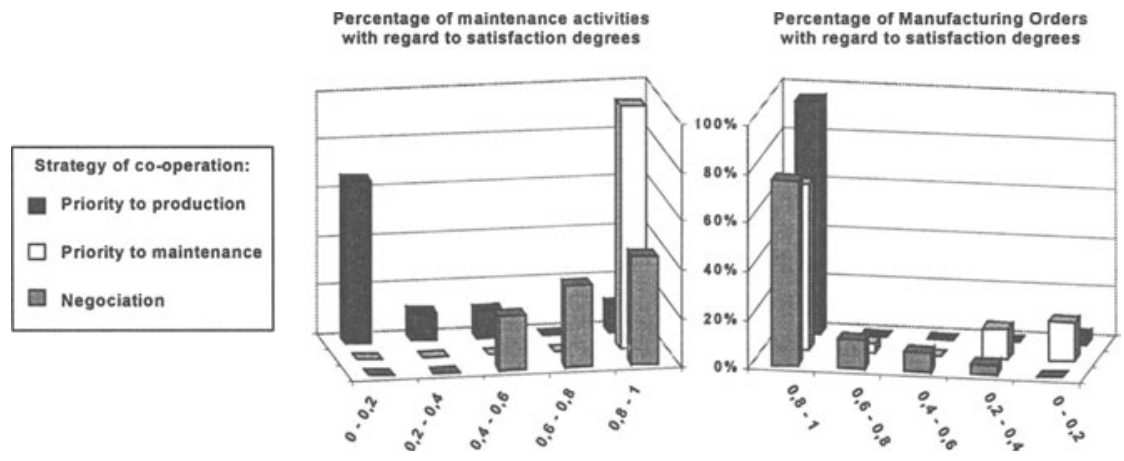

Figure 8 - Results assessment

\section{REFERENCES}

Archimède B, Coudert T. A Multi-Agent Scheduling Approach for the Flexible Manufacturing Production Systems. IFAC Workshop on Distributed Computer Control Systems, DCCS'98, Como, Italy, September 9-11, 1998.

Brandolese M, Franci M, Pozzetti A. Production and maintenance integrated planning. International Journal of Production research, vol. 34, $n^{\circ} 7,1996$.

Coudert T, Archimède B, Grabot B. Un système multi-agents pour la coopération Production/maintenance. Actes de la deuxième conférence francophone de Modélisation et SIMulation (MOSIM'99), Eds : Georges Habchi and Alain Haurat, Publications of SCS International, Annecy, October 6-8, 1999.

Erschler J, Roubellat F, Vernhes JP. Finding some essential characteristics of the feasible solutions for a scheduling problem. Operations Research, $\mathrm{n}^{\circ} 24,1976$.

Ishii H. Scheduling Problems with Fuzzy Constraints. in Scheduling Under Fuzziness, R. Slowinski and M. Hapke Eds, Physica-Verlag, Heidelberg, 2000.

Kerr RM, Walker RN. A Job Shop Scheduling System based on Fuzzy Arithmetic. In Proceedings of the 3rd International Conference on Expert Systems and the Leading Edge in Production and Operation Management. Hilton Head Isl., South Carolina, USA, 1989.

Saad A, Kawamura K, Biswas G. Performance Evaluation of Contract Net-Based Heterarchical Scheduling for Flexible Manufacturing Systems. International Journal of Automation and Soft Computing, Special Issue on Intelligent Manufacturing Planning and Shopfloor Control, 1996.

Sanmarti E, Espuna A, Puigjaner L. Batch production and preventive maintenance scheduling under equipment uncertainty. Computer Chemical Engineering, vol. 21, $\mathrm{n}^{\circ} 10,1997$.

Schăfer K, Patriti V, Charpentier P, Martin P, Spath D. The Multi-Agent Approach in Scheduling and Control of Manufacturing Systems. Proceedings of the Firs International Conference and Exhibition on the Practical Application of Intelligent Agents and Multi-Agents Technology, The Westminster Central Hall, London, 22-24 April, 1996.

Smith RG. The Contract Net Protocol. The International Journal of Flexible Manufacturing Systems, 2, 1990.

Sohier C, Denis B, Lesage J.J. Eco-problem solving for the adaptive control of a production system: the CASPER project, 9th symposium on information control in manufacturing, Nacy-Metz, France, June 24-26, 1998.

Tranvouez E, Espinasse B, Chirac JP. A Multi-Agent Based Scheduling System: a Co-operative and Reactive Approach. 9th Symposium on Information Control in Manufacturing, Nancy-Metz, France June 22-24, Advances in Industrial Engineering, vol. 3, IFAC, 1998. 